\title{
Design and Validation of a Rehabilitation Robotic Exoskeleton for Tremor Assessment and Suppression
}

\author{
E. Rocon, J. M. Belda-Lois, A. F. Ruiz, M. Manto, J. C. Moreno, and J. L. Pons
}

\begin{abstract}
Exoskeletons are mechatronic systems worn by a person in such a way that the physical interface permits a direct transfer of mechanical power and exchange of information. Upper limb robotic exoskeletons may be helpful for people with disabilities and/or limb weakness or injury. Tremor is the most common movement disorder in neurological practice. In addition to medication, rehabilitation programs, and deep brain stimulation, biomechanical loading has appeared as a potential tremor suppression alternative. This paper introduces the robotic exoskeleton called WOTAS (wearable orthosis for tremor assessment and suppression) that provides a means of testing and validating nongrounded control strategies for orthotic tremor suppression. This paper describes in detail the general concept for WOTAS, outlining the special features of the design and selection of system components. Two control strategies developed for tremor suppression with exoskeletons are described. These two strategies are based on biomechanical loading and notch filtering the tremor through the application of internal forces. Results from experiments using these two strategies on patients with tremor are summarized. Finally, results from clinical trials are presented, which indicate the feasibility of ambulatory mechanical suppression of tremor.
\end{abstract}

Index Terms-Human-robot interface, orthotic tremor suppression, rehabilitation robotics, tremor.

\section{INTRODUCTION}

$\mathbf{T}$ HE SCIENTIFIC community is becoming more and more interested in rehabilitation robotics. From a robotic perspective, wearable robots are mechatronic systems worn by a person in such a way that the physical interface permits a direct transfer of mechanical power and exchange of information. A wearable robot is designed to match the shape and function of the human body. Segments and joints correspond to some extent to those of the human body while the system is externally coupled to the person. Initially, the primary applications of these robotic mechanisms were teleoperation and power amplification. Later, exoskeletons have been considered as rehabilitation

Manuscript received September 27, 2006; revised March 29, 2007; accepted April 15, 2007. The work presented in this paper has been carried out with the financial support from the Commission of the European Union, within Framework 5, specific RTD programme "Quality of Life and Management of Living Resources", Key Action 6.4 "Aging and Disabilities", under Contract QKL6-CT-2002-00536, "DRIFTS-Dynamically Responsive Intervention for Tremor Suppression."

E. Rocon, A. F. Ruiz, J. C. Moreno, and J. L. Pons, are with the Biomedical Engineering Group at Consejo Superior de Investigaciones Científicas, Madrid 28500, Spain (e-mail: erocon@iai.csic.es).

J. M. Belda-Lois is with the Instituto de Biomecánica de Valencia (IBV), Valencia 46022, Spain.

M. Manto is with Hôpital Erasme, Brussels 1070, Belgium.

Color versions of one or more of the figures in this paper are available online at http://ieeexplore.ieee.org.

Digital Object Identifier 10.1109/TNSRE.2007.903917 and assistive devices for disabled or elderly people by means of upper and/or lower limb orthosis. One important and specific feature of wearable robotics is the intrinsic interaction between human and robot. This interaction is twofold: first, cognitive, because the human controls the robot while it provides feedback to the human; secondly, a biomechanical interaction leading to the application of controlled forces between both actors.

On the one hand, a typical example of the cognitive interaction is the one being developed through the EMG control of robotic prostheses. Here, the human myoelectrical signals are used to develop control commands to drive an intelligent prosthesis. Force feedback can be implemented by a number of means. On the other hand, a classical example of biomechanical interaction is the exoskeleton based functional compensation of human gait. Here, the robotics exoskeleton applies functional compensation by supporting human gait, i.e., by stabilizing the stance phase. The fact that a human being is an integral part of the design is one of the most exciting and challenging aspects in the design of biomechatronics wearable robots. It imposes several restrictions and requirements in the design of this sort of devices.

Tremor is characterized by involuntary oscillations of a part of the body. The most accepted definition is as follows: "an involuntary, approximately rhythmic, and roughly sinusoidal movement" [1]. Tremor is a disabling consequence of a number of neurological disorders. Although the most common types of tremor were subject to numerous studies, their mechanisms and origins are still unknown. Tremor, the most common of all involuntary movements, can affect various body parts such as the hands, head, facial structures, tongue, trunk, and legs. Most tremors, however, occur in the hands.

Tremor is a disorder that is not life-threatening, but it can be responsible for functional disability and social inconvenience. More than $65 \%$ of the population with upper limb tremor have serious difficulties performing daily living activities [2], [3]. In many cases, tremor intensities are very large, causing total disability to the affected person. The overall management of tremor is directed towards keeping the patient functioning independently as long as possible while minimizing disability.

It has been established in the literature that most of the different types of tremor respond to biomechanical loading [4]-[6]. In particular, it has been clinically tested that the increase of damping and/or inertia in the upper limb leads to a reduction of the tremorous motion [4], [6]. This phenomenon gives rise to the possibility of an orthotic management of tremor. An orthosis is a wearable device that acts in parallel to the affected limb. In the case of tremor management, the orthosis must apply a damping or inertial load to a selected set of limb articulations. As a wearable device, it must exhibit a number of aesthetic and 
functional characteristics. Aesthetics is more directly related to size, weight and appearance of the exoskeleton. Functionality is related to generating required torque and velocity while maintaining the robustness of operation.

In the framework of the DRIFTS (dynamically responsive intervention for tremor suppression) project [7], the WOTAS exoskeleton was presented with three main objectives: monitoring, diagnosis, and validation of nongrounded tremor reduction strategies [8]. This paper presents the development and validation of such a platform. In the next section, the biomechanics of the upper limb is studied. In third section, the description of the WOTAS exoskeleton is given. Next, two novel nongrounded control strategies for suppression of tremor by means of an orthotic (wearable) exoskeleton are presented. Both are based on biomechanical loading, but one is active and the other is passive. 1) Tremor reduction through impedance control. This strategy modifies the stiffness, damping and mass properties of the upper limb in order to suppress tremor. 2) Notch filtering at tremor frequency. This strategy implements an active noise filter at the tremor frequency taking advantage of the repetitive characteristics of tremor. Section IV describes the clinical experiments for system validation. Finally, the conclusions and future work of this study are given.

\section{WOTAS}

An orthosis is defined as a medical device that acts in parallel to a segment of the body in order to compensate some dysfunction. The main function of the arm is to position the hand for functional activities. The hand must be able to reach any point in the space, especially any point on the human body, in such a way, that the person can manipulate, draw on, and move objects to or from the body. Therefore, the kinematic chain formed by the shoulder, elbow, forearm, wrist, and hand, has a high degree of mobility. In this way, the upper limb is one of the most anatomic and physiologically complex parts of the body.

The upper limb is very important because it is able to execute cognition-driven, expression-driven, and manipulation activities. Furthermore, it intervenes in the exploration of the environment and in all reflex motor acts. For this reason, any alteration or pathology that affects the upper limb motion range, muscle power, sensibility, or skin integrity will alter its operation. The concept of WOTAS is to develop an active upper limb exoskeleton based on robotics technologies capable of applying forces to cancel tremor and retrieve kinematic information from the upper limb.

\section{A. Mechanical Design}

WOTAS was developed to provide a means of testing nongrounded tremor reduction strategies. WOTAS follows the kinematic structure of the human upper limb and spans the elbow and wrist joints, see Fig. 4. It exhibits three degrees-of-freedom corresponding to elbow flexion-extension, forearm pronation-supination, and wrist flexion-extension. In the final design, WOTAS restricts the movement of wrist adduction-abduction. This strategy was chosen because this is the upper limb movement with the least impact in daily living activities, [9].
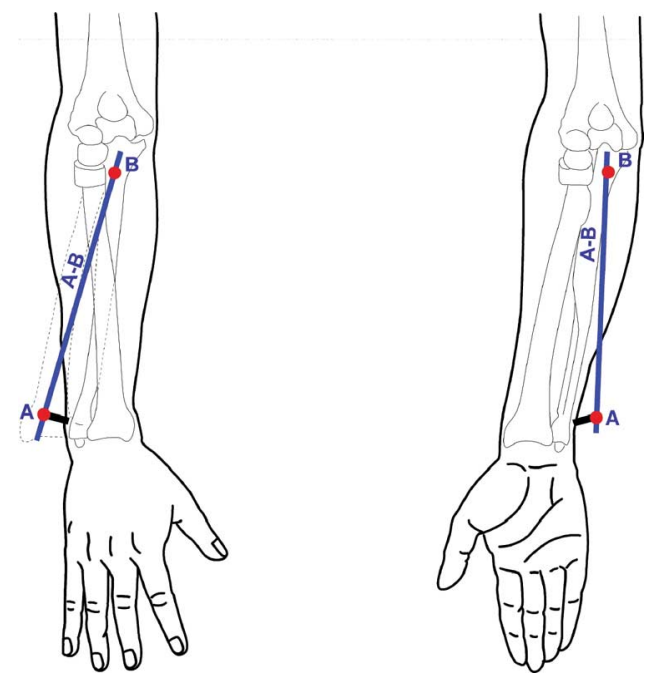

Fig. 1. Scheme of the pronation-supination control.

The mechanical design of the joints for elbow flexion-extension, and wrist flexion-extension are similar to other orthotic solutions and is based upon the behavior of those physiological joints as hinges. For orthotic purposes, flexion-extension movement is considered as a pure rotational movement. Therefore, this axis of rotation should be used for the rotational actuator. The axis of rotation for the elbow joint is placed in the line between the two epicondyles. The axis of rotation for the wrist joint is located in the line between the capitate and lunate bones of the carpus. The mechanical design for the control for the pronation supination movement is more complex and it is explained below.

1) Pronation-Supination Control: The pronation-supination movement of the forearm is a rotational movement of the forearm on its longitudinal axis which engages two joints that are mechanically connected: the upper radioulnar joint (which belongs to the elbow) and the lower radioulnar joint (which belongs to the wrist) [10]. There are two bones in the forearm that make this movement possible.

- The ulna is the bone that remains fixed during the pronation-supination movement. It constitutes the main part of the elbow, in particular the olecranon.

- The radius is the moving bone in the pronation and supination. It rotates in proximal part (close to the elbow) and moves distally along the axis formed by the ulna bone (see Fig. 1).

Both bones have a shape approximately pyramidal and they are placed in such a way that the base of the radius is in the tip of the ulna and vice-versa.

The WOTAS platform controls the pronation-supination movement with the rotation control of a bar parallel to the forearm. This bar is fixed very close to the olecranon (Fig. 1, point $\mathrm{B}$ ). Thus the bar is fixed to the ulnar position at elbow level. The distal fixation of the bar is made at the head of the radius, although the bar is maintained in the ulnar side in order to minimize the excursion of the system. This fixation is explained later in the support design section.

2) Design of the Support System: There are no static orthoses that achieve tremor suppression due to the intrinsically 


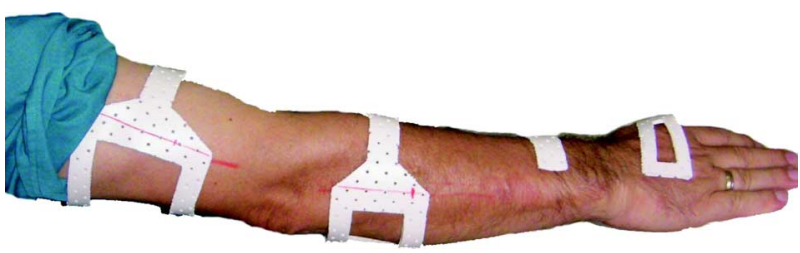

Fig. 2. Structure of WOTAS support.

dynamics characteristics of tremor. In these cases, the tremor suppression mechanism tends to lose their alignment instead of suppressing tremor.

To determine the points of the upper limb where the dynamic forces would be applied, i.e., the points where the arm supports would be placed for the interface between the actuators and the arm, a number of biomechanical and physiological considerations of the upper limb have to be observed, such as [11]: 1) the forces on the arm tissues must stay in acceptable limits; 2) the application of forces on the arm that have minimal interference of elbow and wrist movement; 3 ) the interaction of the robotic device with the arm, i.e., where the forces will be applied on the upper limb and how the load will be transmitted to the person for optimum comfort. To respond to these issues, a biomechanical study was done of the upper limb [11]. The aim of this study was to determine the limits of comfort regarding pressure, so that there is an upper limit to the total force that can be applied safely to the upper limb. This study analyzed two key aspects: the person's perception of the pressure and the maximum pressure tolerance thresholds [11]. The first aspect is important to select the appropriate strategy to apply to the load on the body.

For the development of the mechanical structure, different types of materials for the securing or support elements between the orthotic device and the arm were considered. The mechanical conditions of these elements are critical because they must ergonomically couple the upper limb, and also the rigidity of the material must be greater than the rigidity of the underlying tissues. To securely fix the structure it was decided to use supports made from thermoplastic. With this type of material, supports are obtained that adapt to the morphology of each user's arm, Fig. 2. Each support has at least three contact points per segment and thus misalignments are avoided between the orthosis and limb [12]. Velcro straps were fixed to the fabric in order to tighten the support to the arm. The fixation to the wrist is mainly placed over the radial side for the reason that the wrist follows the movement of the head of the radius. Both the distal tip of the pronation-supination control and the proximal tip of the wrist control are fixed to the ulnar side of the wrist. The fixation to the hand is very similar to that of the wrist. In addition, a textile substrate was used to compress the soft tissues and enhance performance of the fixation supports.

\section{B. Sensors}

The system aims to allow both monitoring of tremor data and implementation of tremor suppression strategies. Therefore, it is equipped with kinematic (angular velocity) and kinetic (interaction force between limb and orthosis) sensors.

Tremor force, position, velocity, and acceleration are the required information to implement the two control strategies. The

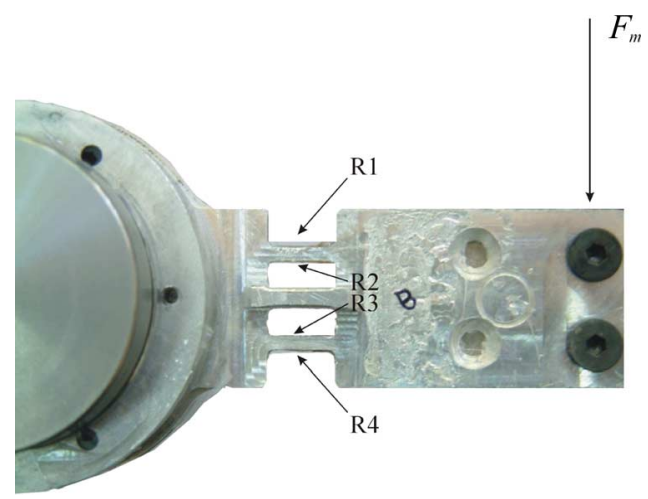

Fig. 3. Full Wheatstone bridge and gauges (R1, R2, R3, R4) mounted on WOTAS structure.

types of sensors were restricted to the following sensors: goniometry, gyroscopes, and accelerometers. MEMS gyroscopes were selected as a promising technology. The main advantages of using gyroscopes are [13]: they measure rotational motion (human motion is rotational about joints), they are not influenced by gravity, both frequency and amplitude information accurate down to direct current (dc) (zero frequency), based on mathematical operations it is possible to obtain angular displacement and acceleration, high signal-to-noise ratio, high dynamic range, and solid state gyros do not influence motion of subject being measured.

An analysis of commercial solid-state gyros was performed. Two alternatives from the Japanese manufacturer Murata were selected: the GYROSTAR ENC-03J and its surface mounted device (SMD) version, and the GYROSTAR ENC-03M.

Since gyroscopes provide absolute angular velocity in its active axis, the combination of two independent gyroscopes, placed distallly and proximally to the joint of interest, is required. In order to perform the required treatment of the sensor output to integrate them into WOTAS architecture some electronics have been developed: a band pass filter with a low cutoff frequency of $0.3 \mathrm{~Hz}$ in the sensor output and a higher cutoff frequency of $25 \mathrm{~Hz}$. This is the frequency band we considered relevant to the application [3]. The concept of using gyroscopes as an ungrounded method to assess tremor variables was evaluated and approved. The system is light, portable, small, inexpensive, and does not lead any discomfort to subjects [14].

Since no backdrivable actuators were chosen for the application (see next section), it was also decided to use force sensors as a means of implementing impedance feedback control strategies. Strain gauges in a full Wheatstone bridge have been selected as force sensors. The gauges measure the torque applied by the motors on the WOTAS structure, therefore, they are mounted on the structure so that they only measure the force perpendicular to the motor axis $\left(F_{m}\right)$, thus their measurement is not affected by forces caused in undesired directions, Fig. 3. The extensiometric gauges are connected to a Wheatstone Bridge circuit in a combination of four active gauges (full bridge).

The strain gauge system was characterized and the sensitivities of the system were derived in the three planes. The tests performed showed that the system has a very low sensitivity to 
orthogonal forces. The system presented a thermal drift, however, after a few minutes the output voltage becomes stable and suitable for operation.

\section{Actuators}

Before the selection of specific actuators to suppress tremor, an estimation of the required torque and power was performed. This was achieved through an analysis of kinematic tremor data obtained from a study performed by the authors [11]. From this torque estimation of effort that the exoskeleton structure must support, duralumin was selected as the material to construct the exoskeleton structure. This material was selected in order to build a lightweight structure with sufficient rigidity to support the efforts.

Based on this study, a number of candidate actuators were evaluated [12]. The analysis was restricted to the following actuators: electro active polymers (EAPs), electro and magneto rheological fluids (ERF-MRF), dc motors, shape memory actuators (SMAs), pneumatic muscles, and ultrasonic motors.

The actuator technology should have a high-power density allowing the implementation of a compact and light solution suitable for wearable devices. Based on this criteria, both dc motors and ultrasonic motors can be regarded as best alternatives for exerting tremor suppression forces. The former are well-known off the-shelf technologies of easy integration in advanced control schemes but bulky. The latter are less flexible but offer very compact solutions due to their dynamic range (low velocity high torque motors). Both alternatives were evaluated in prototypes and dc motor was the technology selected for the final version of WOTAS. The main problem with the ultrasonic motors was their poor response at low speed, therefore, leading to problems to track user's slow voluntary motion [15].

Owing to the problems encountered with ultrasonic motors, a new WOTAS device was constructed using dc motors as an actuation element. The dc motor selected to activate WOTAS articulations was a Maxon dc flat brushless motor EC45. In order to match the speed and torque of the dc motor to the application requirements, a harmonic transmission drive was used. In particular, the drive selected for our application was the HDF-014-100-2A. This configuration, based on flat dc motors and pancake transmissions, is able to provide a maximum torque of 8 N.m, nevertheless the maximum torque was electronically limited to $3 \mathrm{~N} . \mathrm{m}$ in order to guarantee the safety of the user. Fig. 4 illustrates the final configuration of WOTAS system activated by dc motors.

The total weight of the final system is roughly $850 \mathrm{~g}$. A protocol for testing the system was performed to evaluate the usability and the range of workspace allowed to a normal user. The system was used in the laboratory to perform a wide variety of manoeuvres in free mode. These preliminary tests successfully showed the correct operation of the system and the capability of the system to access the workspace, without affecting the normal range of motion of the user [8].

\section{Control Architecture}

The WOTAS control architecture basically consists of three components: 1) the exoskeleton, with its structure, sensors and
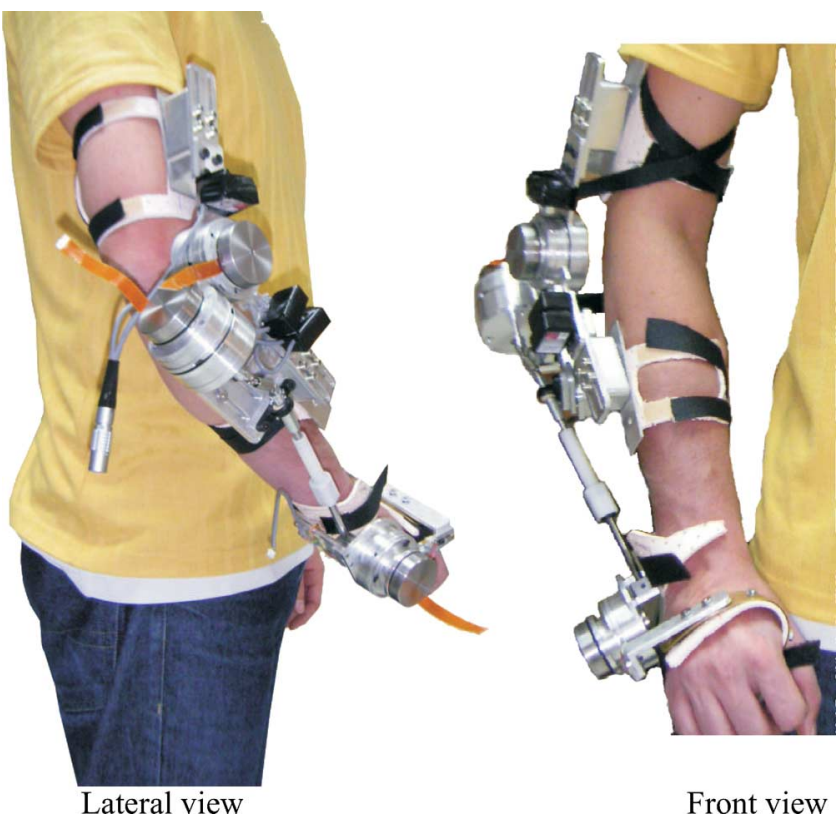

Fig. 4. Final version of WOTAS for the control of three human upper-limb movements: flexion-extension of the elbow, flexion-extension of the wrist and pronation-supination of the forearm.

actuators; 2) a control unit, responsible for executing the algorithms in real time to suppress the tremor and the acquisition card for the interface between the sensors, actuators and the controller; 3) a remote computer, which in our case executes an application developed for the interface between the system and the doctor who is using it.

This control architecture interfaces the control algorithms with the orthosis. The WOTAS circuitry and sensors serve two functions: 1) to obtain the position, angular velocity and acceleration signals needed for control, data collection, and evaluation and 2) to generate the power signal to activate the actuator.

The control of the entire active orthosis was implemented in the MatLab RT environment. This environment provides mathematically complex control strategies in real time. The interface between the MatLab environment and the active orthosis is based on a standard data acquisition board.

In order to provide an interface to all the control strategies, a software application was developed in C language. It communicates with the low level controller (either by TPC/IP, wired serial link or BlueTooth) using Dynamic Link Libraries (DLLs) developed by the authors [16]. This interface monitors signals and tune controller parameters in real time during the control strategy execution. It has five main objectives: 1) monitoring and validation of algorithms for tremor suppressionl; 2) data analysis (statistics, algorithms performance, etc.); 3) storage of user information such as clinical and anthropometrics data; 4) comparison between different control strategies; 5) extraction of user parameters: joint position, velocity, and acceleration; tremor frequency (algorithm implemented in the Target PC); tremor torque; and power (based on an upper limb biomechanical model) [11].

It was also possible to save all the information retrieved by the sensors for future offline analysis. 


\section{CONTROL STRATEGIES FOR TREMOR SUPPRESSION}

The approach to suppress the pathological tremor within this study is to assist the limb with compensatory technology in order to decrease the amplitude of tremor. Briefly, the control system should work as follows: sensors coupled to the limb measure its motion, an error cancelling algorithm performs a real-time discrimination of the undesired component of motion, tremor information is input to the controller in order to generate the desired actuator action to suppress the tremor. This concept can be approached either by ambulatory orthotic devices or nonambulatory solutions among which we can consider wheelchair mounted devices. The former approach is characterized by selective tremor suppression through internal forces at particular joints, while the latter relies on a global application of external forces that leads to overall tremor reduction. Both ambulatory and nonambulatory concepts for tremor suppression can be found in the literature. Furthermore, both concepts can be implemented through passive and active systems, [3], [5].

In active concepts, the system generates an equal but opposite motion to the tremor, actively compensating and effectively subtracting the tremor from the overall motion. This force is generated by the system's motors as a result of a control algorithm. In passive concepts, a mechanical damper is used, thus the dissipative force usually results from viscous friction or inertia provided by the damper [5].

One of the main drawbacks of the systems described in literature is that the dissipative force is also loading the patient's voluntary motion. As a consequence, the user feels a mechanical resistance to the motion. Even though in adaptive systems this could be avoided, filtering out the voluntary motion eliminates the resistance to the voluntary motion. For a successful adaptive tremor absorption mechanism, a means for intelligent detection of tremor versus voluntary motion is required. To this end, a model of the tremor motion is proposed in next section. Two novel control approaches were developed, one passive and one active, based on biomechanical loading for tremor suppression by means of wearable exoskeletons [17].

- Tremor reduction through impedance control-implements an impedance control, i.e., the stiffness, damping, and mass properties of the upper limb can be modified to study its effects on tremor.

- Notch filtering at tremor frequency-based on noise reduction techniques and implements an active noise filter at the tremor frequency.

\section{A. A Model of Tremor}

In addition to being important to distinguish between desired and undesired motion, the analysis of the tremor signal, both in terms of frequency and amplitude, is relevant to assess the stationary characteristics of tremor, i.e. frequency drift and amplitude variation. This information is important when designing control strategies to counteract tremor.

A number of estimation algorithms have been developed for tremor suppression. As a first approach, we used robust algorithms based on IEEE-STD-1057, which is a standard for fitting sine waves to noisy discrete-time observations. In particular, the weighted-frequency Fourier linear combiner (WFLC) developed by Riviere [18] in the context of actively counteracting physiological tremor in microsurgery was implemented. The WFLC is an adaptive algorithm that estimates tremor using a sinusoidal model, estimating its time-varying frequency, amplitude, and phase. The WFLC can be described by (1). It assumes that the tremor can be mathematically modelled as a pure sinusoidal signal of frequency $\omega_{0}$ plus $M$ harmonics and computes the error $\varepsilon_{k}$ between the motion $s_{k}$ and its harmonic model

$$
\varepsilon_{k}=s_{k}-\sum_{r=1}^{M}\left[w_{r_{k}} \sin \left(r \omega_{0_{k}} k\right)+w_{r+M_{k}} \cos \left(r \omega_{0_{k}} k\right)\right] \text {. }
$$

In its recursive implementation, see (2) and (3), the WFLC can be used online to obtain estimations of both tremor frequency and amplitude [19]

$$
w_{0_{k+1}}=w_{0_{k}}+2 \mu_{0} \varepsilon_{k} \sum_{r=1}^{M} r\left(w_{r_{k}} x_{M+r_{k}}-w_{M+r_{k}} x_{r_{k}}\right)
$$

where

$$
x_{r k}=\left\{\begin{array}{ll}
\sin \left(r \sum_{t=1}^{k} w_{0_{t}}\right), & \text { for } 1 \leq r \leq M \\
\cos \left((r-M) \sum_{t=1}^{k} w_{0_{t}}\right), & \text { for } M+1 \leq r \leq 2 M
\end{array} .\right.
$$

The WFLC algorithm was evaluated in signals measured in patients suffering tremor, [12]. In the completed trials, the algorithm was able to estimate the tremor movement of all the patients with accuracy always lower than $2^{\circ}$. The main disadvantage of the WFLC is the need for a preliminary filtering stage to eliminate the voluntary component of the movement [19]. This filtering stage introduces an undesired time lag for our system when estimating tremor movement, this time lag introduces a time delay that could considerably affect the implementation of the control strategies for tremor suppression.

The solution adopted was the development of an algorithm capable of estimating voluntary and tremorous motion with a small phase lag. The tremor literature [2], [18], [20], indicates that voluntary movements and tremor movements are considerably different. Voluntary movements are slower while tremor movements are brusquer. This indicates that adaptive algorithms to estimate and track movement would be useful when separating the two movements with an appropriate design. The underlying idea is to design the filters so that they only estimate the less dynamic component of the input signal, which in our case we consider to be voluntary movement, thereby filtering out the tremor movement. Thus, to estimate voluntary movement and tremor movement, the development of a two-stage algorithm is proposed to estimate voluntary movement and tremor movement with a minimum time lag, see Fig. 5.

In the first stage, a set of algorithms was considered for the estimation of the voluntary motion: two point-extrapolator, critically damped $g-h$ estimator, Benedict-Bordner $g-h$ estimator, and Kalman filter. These algorithms implement both estimation and filtering equations. The combination of these actions allows the algorithm to filter out the tremorous movement from the overall motion at the same time it reduces the phase lag introduced, [21]. The equation parameters were adjusted to track the movements with lower dynamics (voluntary movement) since 


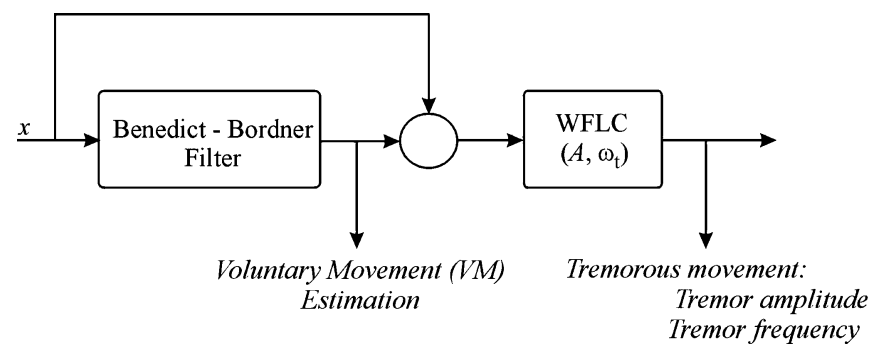

Fig. 5. Two-stage tremor modelling: first, the low frequency content voluntary motion is estimated, secondly, the voluntary motion estimation is subtracted from the original motion, eventually, tremor frequency and amplitude are determined.

tremors present a behavior characterized by quick movements [12].

The algorithms evaluated were two degrees of freedom estimators i.e., they assume a constant velocity movement model. This assumption is reasonable since the sample period is very small compared to the movement velocities [22], i.e., the sample period adopted was $1 \mathrm{~ms}$ and the voluntary movement estimated occurs in a bandwidth lower than $2 \mathrm{~Hz}$. The performance of these algorithms were compared based on their accuracy when estimating voluntary movements of tremor time series from patients. The result of such analysis indicated that Benedict-Bordner filter presents the best results with the lowest computational cost, [12]. This estimation algorithm is a $g-h$ filter with the following tracking update equations:

$$
\begin{aligned}
\dot{x}_{k, k}^{*} & =\dot{x}_{k, k-1}^{*}+h_{k}\left(\frac{y_{k}-x_{k, k-1}^{*}}{T}\right) \\
x_{k, k}^{*} & =x_{k, k-1}^{*}+g_{k}\left(y_{k}-x_{k, k-1}^{*}\right)
\end{aligned}
$$

and by $g-h$ prediction equations [22]

$$
\begin{aligned}
\dot{x}_{k+1, k}^{*} & =\dot{x}_{k, k}^{*} \\
x_{k+1, k}^{*} & =x_{k, k}^{*}+T \dot{x}_{k+1, k}^{*} .
\end{aligned}
$$

The tracking update equations or estimation equations [(4) and (5)] provide the joint angular velocity and position. The estimated position is based on the use of the actual measurement as well as the past prediction. The estimated state contains all the information we need from the previous measurements. The predicted position is an estimation of $x_{n+1}$ based on past states and prediction, (6) and (7), and take into account the current measurement by means of updated states. The Benedict-Bordner estimator is designed to minimize the transient error. Therefore, it responds faster to changes in movement velocity and it is slightly under-damped [21]. The relation between filter parameters are defined by (8)

$$
h=\frac{g^{2}}{2-g} .
$$

In the second stage, the estimated voluntary motion is removed from the overall motion and it is assumed that the remaining movement is tremor. After this, we use the WFLC in order to estimate tremor parameters. In this stage, the algorithm estimates both the amplitude and the time-varying frequency of the tremorous movement.

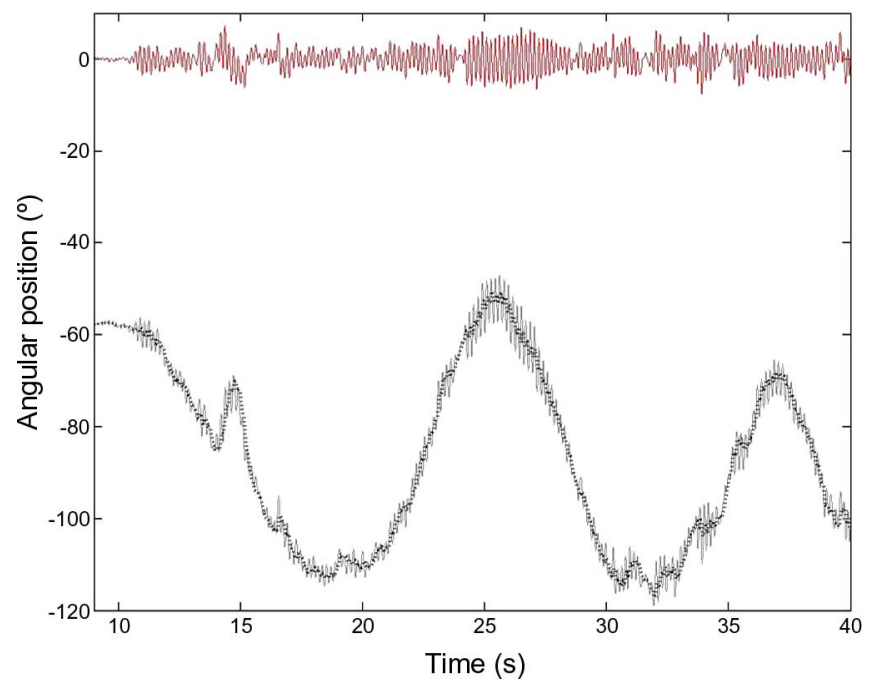

Fig. 6. Modelling of tremor as a sinusoidal non-voluntary motion: velocity signals (gray) obtained from gyroscopes, estimation of voluntary movement (black) and estimation of tremorous movement (red).

This algorithm was evaluated with data obtained from 40 patients suffering from different tremor diseases. The estimation error of the first stage was $1.4 \pm 1.3^{\circ}$ [12]. The second stage algorithm has a convergence time always smaller than $2 \mathrm{~s}$ for all signals evaluated and the mean square error (MSE) between the estimated tremor and the real tremor (obtained offline by means of manual decomposition based on classical filter techniques), after the convergence, is smaller than $1^{\circ}$. The combination of both techniques resulted in a very efficient algorithm with small processing cost for estimating in real time the voluntary and the tremorous components of the overall motion [12]. Fig. 6 illustrates the performance of the algorithm when splitting voluntary and tremorous movements of a patient of essential tremor.

\section{B. Tremor Reduction Through Impedance Control}

The impedance of a system comprises three components, i.e., stiffness, damping and inertia [23]. There is evidence [6] that all three components modify the biomechanical characteristics of tremor at the upper limb, which in general, can be described by a second-order system [6], [24].

Our approach consists in changing the biomechanical characteristics of the musculo-skeletal system by means of selecting the appropriate modified value of damping and inertia of the musculo-skeletal system in order to reduce the amplitude of the tremorous movement, see Fig. 7. Unlike other approaches in the literature, the control scheme is conceived so that the effect of the suppression load on voluntary motion is minimized.

The control approach is based on a dual control loop, Fig. 7. The value of force applied by the exoskeleton over the upper limb, $\tau_{d}$, is calculated based on the summation of the effects of both loops

$$
\tau_{d}=f_{d t}-f_{m t}-\tau=f_{d t}-k_{i} \ddot{q}_{t}-k_{v} \dot{q}_{t}-\tau \text {. }
$$

This closed-loop control architecture uses the information from the gyroscopes $\dot{q}$ on the data treatment block to distinguish between voluntary and tremor motion, $\dot{q}_{t}$, from the overall 


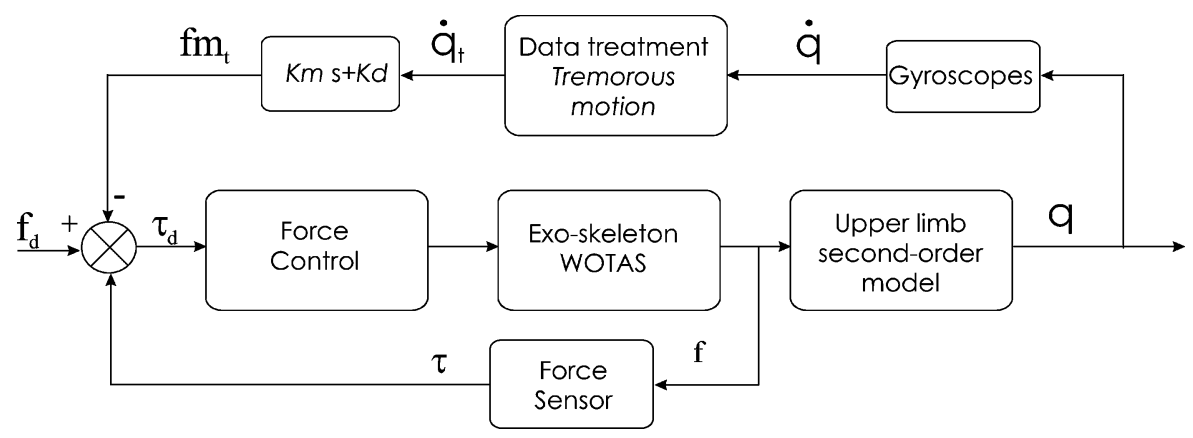

Fig. 7. Control strategy to modify the biomechanical parameters of the upper limb.

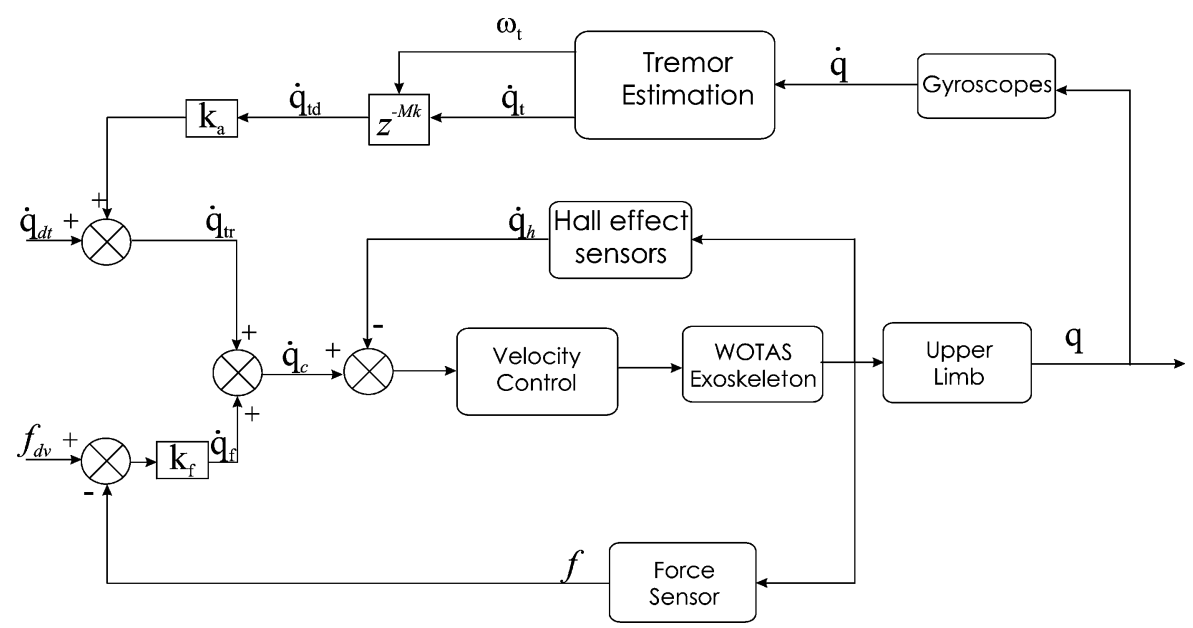

Fig. 8. Active tremor suppression control strategy.

movement (as explained in the previous section). The angular velocity information from the estimated tremorous component, $\dot{q}_{t}$, is subsequently multiplied by the coefficients $K_{m}$ and $K_{d}$, which describe the reference inertia and damping characteristics of the upper limb. This process defines the actual impedance force, $f m_{t}$, of the system which is set to reduce the tremor. This impedance force should tend to vanish as tremorous motion is suppressed.

The lower loop of Fig. 7 serves the goal of minimizing the effect of the orthosis on the voluntary motion. In this case, force sensors measure the interaction force between orthosis and the limb, $f$. Under ideal circumstances, a user free of pathologic tremor should feel no force from the orthosis, i.e., no loading should be a consequence of voluntary motion. In order to achieve this, the interaction force $f$ is filtered so that just the force opposing voluntary motion $\tau$ is fed back in the lower branch of the control loop.

The control strategy proposed has an adaptive behavior so that constantly (in real time) it updates the tremor amplitude estimate. Thus the system can respond to the changes produced by the control strategy on the tremor, [25].

\section{Notch Filtering at Tremor Frequency}

Tremor frequency varies according to the particular neurological disorder being considered. In particular, while essential tremor takes place in the frequency range between 5 and $8 \mathrm{~Hz}$, rest tremor is usually found at a slightly lower frequency range, 3-6 Hz.

In addition, for a given type of tremor, its main frequency varies from patient to patient, but tends to be quite stable for a particular subject. This property should be exploited when designing a control strategy to counteract tremor. In particular, repetitive control can handle periodic (repetitive) signals and disturbances. Repetitive control can be regarded as a subset of learning control since the control action is determined using the stored error values from preceding periods. Even though repetitive approaches can handle periodic signals (tremor), it is not free from some common problems: tight stability conditions, poor response to nonperiodic and nonharmonic signals and poor noise characteristics. As reported by Inoue [26], a stabilizing compensation and smoothing of the control signal over periods can be used to overcome the above mentioned problems.

The control strategy designed and proposed for active control of the pathological tremor manages the exoskeleton to generate a motion equal but opposite to the tremor, based on the real time estimation of the involuntary component of motion. The control strategy that controls the velocity of each articulation of WOTAS is illustrated in Fig. 8.

As illustrated in Fig. 8, this control strategy is also composed by a dual control loop. The upper control loop is responsible for tremor suppression while the lower control loop should minimize the influence of the control strategy on the voluntary movement, by means of an admittance control [12]. 
In the upper loop, the algorithm presented in Section III-A estimates the angular velocity of tremorous movement, $\dot{q}_{t}$. This value is delayed by $M$ seconds. $M$ is defined by (10), where $\omega_{s}$ is the sample frequency of the control strategy and $\omega_{t}$ is the estimated tremor frequency

$$
M=\omega_{s} / \omega_{t} .
$$

The velocity amplitude of the tremorous movement delayed by $M$ samples is fed-back to the inner velocity control loop of WOTAS exoskeleton. This makes the orthosis replicate the velocity amplitude of the tremorous movement but opposite to the tremor, implementing a notch filter at tremor frequency and actively compensating the tremorous movement of the user [12]. The following equation define the upper control loop of the active strategy:

$$
\dot{q}_{c}=\dot{q}_{f}+\dot{q}_{t r}=k_{f}\left(f_{d v}-f\right)+\left(\dot{q}_{d t}+z^{-M_{k}} \cdot \dot{q}_{t}\right) .
$$

As in the previous control approach, the quality of the tremor suppression control approach is strongly dependent on accurate estimation and tracking of tremor frequency. In [12] it is shown that the system is stable when the algorithm for tremor estimation is stable.

In summary, the operation of WOTAS system for tremor suppression is based on the identification of tremorous motion out of tremorous motion measured by kinematic and kinetic sensors. Adaptive algorithms help identify and distinguish tremorous motion from the voluntary one. This information is then used to establish a proper physical interaction (modification of the combined human-exoskeleton articular impedance or applying forces opposite to tremor) that should result in tremor reduction.

Another very important characteristic in the implementation of the proposed control strategies is that the control strategy proposed is based on an articular control approach because it is simpler and also makes it possible to implement individual control loops in each joint with a high dynamic range [27]. Furthermore, the fact that each exoskeleton joint tries to suppress the tremor generated in its corresponding anatomical joint is interesting because it guarantees reducing the tremor in each joint. Thus, the problem of coupling the tremor between the upper-limb joints is successfully tackled [8].

In studies done by the authors, the behavior and contribution of each joint in the upper-limb tremor were evaluated, [25]. This work has shown that in most patients the tremor movement "displaces" along the kinematic chain of the arm when its effects are reduced (by applying biomechanical loads) on one of the arm joints. However, the study of tremor behavior when its effects are cancelled in different joints of the arm has still not been properly studied [3]. This aspect led to devising active and independent control strategies in each joint. Accordingly, if the cancellation of the tremor in one of the joints increases the tremor in the other joint, the algorithm responsible for controlling the adjacent joint will identify the increased tremor and try to reduce the tremor generated by coupling the upper-limb joints. The aim is thus that the active behavior of tremor reduction in each joint reaches equilibrium, thereby decreasing the coupling effects of the upper-limb joints. In addition, the range of move- ment of each articulation of the exoskeleton is limited by the control strategies in such a way that they never exceeds the natural range of motion of the user. This was implemented in order to guarantee user safety.

\section{EXPERIMENTAL PROTOCOL}

In order to evaluate the performance of the device developed to suppress tremor, we conducted an experimental phase involving 10 patients suffering from different tremor diseases. These experiments were conducted in Hôpital Erasme, in Belgium, and Hospital General Universitário de Valencia, in Spain, and were led by a neurologist. The protocol, the results, and the analysis of the results are presented in the following sections.

\section{A. Users}

Ten users participated in these experiments (three women, mean age 52.3 years). Users presented different pathologies but the majority was affected by essential tremor (ET). ET was moderate in users 1,3,4, and 7 and severe in users 2, 5, and 6. User 8 suffer from multiple sclerosis, user 9 from posttraumatic tremor and user 10 is affected by a mixed tremor. All users provided their informed consent. The investigation was approved by the ethical committee of both hospitals. All the experiments were recorded.

The users still exhibited tremor despite a regular intake of the drugs conventionally administered for tremor, even at high doses for some of the users.

\section{B. Materials and Methods}

Three different people were present during the measurements.

- A computer operator: In charge of setting the parameters of the systems and recording the signals.

- A medical doctor: In charge of supervision of the condition of the trials and the state of the user.

- An experimenter: In charge of fitting and removing the orthosis and interacting with the user to perform the trials.

During the experiments neither the user, nor the experimenter or the medical doctor knew when the systems were applying a suppressing strategy or when it was operating in monitoring mode. Just the computer operator knew when the systems were applying the suppression strategy. For formal purposes, we consider this arrangement equivalent to a double-blind trial in order to reduce the placebo effects in the experimentation phase [28].

\section{Tasks}

Three different tasks were selected to be performed by the users: keep the arm outstretched, point the nose with a finger, and keep the arm in a rest position. These tasks have been previously used to characterize tremor movement [28].

During the experiments, WOTAS operated basically in its three different control modes as follows.

1) Monitoring mode. WOTAS operate in free mode (no force is applied on the upper limb) and monitor tremor parameters of the users.

2) Passive Control mode. WOTAS is able to change biomechanical characteristics of upper limb, such as viscosity or inertia, in order to suppress tremor (Section III-B). 
3) Active Control mode. WOTAS is able to apply opposite forces to the tremorous movement based on a real time estimation of the involuntary component of motion (Section III-C).

The order in which the modes have been applied has been alternated, as well as the order in which the users have executed the tasks. In each experimental session, three repetitions of each task were realized. This approach was adopted in order to avoid interactions in the analysis, as well as learning effects [28]. The number of repetitions has been chosen in order to have an experimental session not longer than $1 \mathrm{~h}$.

\section{Data Analysis}

The data analyzed were the output voltage coming from the gyroscopes placed on the active orthosis. This output voltage was sampled at a $2000 \mathrm{~Hz}$ rate. The data has been filtered using a Kernel Smoothing algorithm and a gaussian window 51 points width. The figure of merit adopted to quantify the reduction achieved by the exoskeleton is the ratio between the signal analyzed in monitoring mode $\left(P_{m m}\right)$, and the signal analyzed in suppression mode $\left(P_{s m}\right)$, both in passive or active modes, (12). Therefore, the reduction of tremor was measured with the users under the same conditions: with the orthosis placed on the upper limb. As a result, the estimated reduction was the remaining tremor in suppression mode referred to tremor at monitoring mode

$$
R=\frac{P_{s m}}{P_{m m}} \cdot 100 .
$$

The parameter selected to compare the tremor level is the Power contained in the frequency band from 2 to $8 \mathrm{~Hz}$ [3]. It can be defined as follows:

$$
\begin{aligned}
\Phi(f) & =\frac{F F T(x) F F T(x)^{*}}{N} \\
P & =\sum_{i=f_{1}}^{f_{2}} \frac{\Phi(i)}{T}=\frac{1}{N t_{s}} \sum_{i=f_{1}}^{f_{2}} \Phi(i)
\end{aligned}
$$

where $x$ is the signal in the time domain, $N$ is the length of the signal, $t_{s}$ is the sampling period and, $f_{1}$ and $f_{2}$ are the lower and upper limits of the range of interest.

\section{E. Results and Discussion}

The effects of adding effective viscosity were investigated for the upper limb during the execution of the different tasks. During the trials, some users were able to identify when the system was operating in suppression mode, relating to the clinician either "now the system is suppressing my tremor" or "now it is not."

Fig. 9 illustrates the performance of WOTAS when operating in suppression mode for all subjects in this experiment. Notice that the efficiency of the exoskeleton improves with tremor power increase. An statistical analysis has been made to characterized the tremor suppression. The statistical analysis has been made using R [29]. A second-order polynomial fit has been made with the natural logarithms of power spectra in free and suppression mode, see red line on Fig. 10. As showed in Table I,

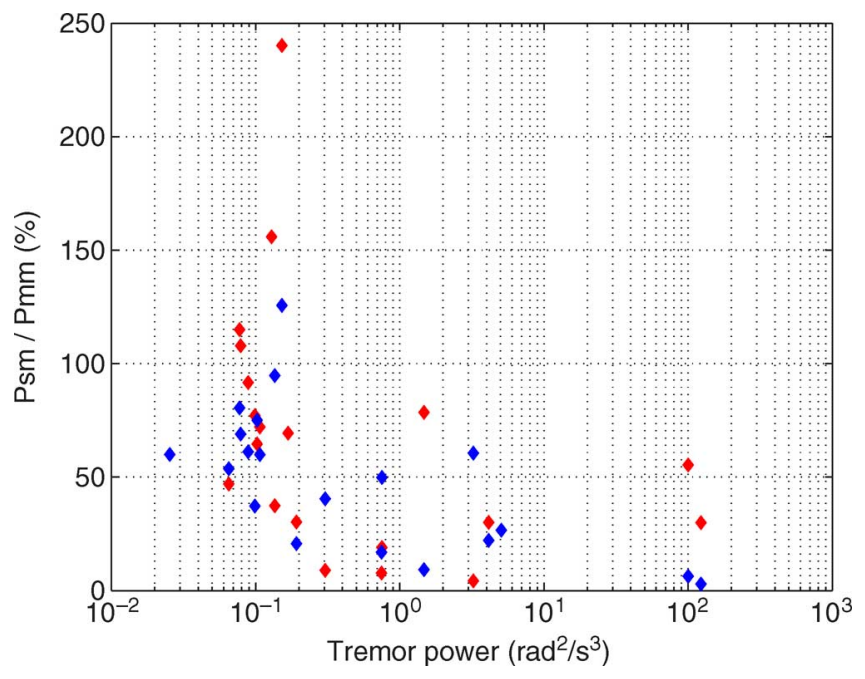

Fig. 9. Tremor reduction ( $y$ axis) achieved by WOTAS operating in suppressing both in active (blue markers) and passive (red markers), mode. $x$ axis represents users' tremor energy with WOTAS in monitoring mode.

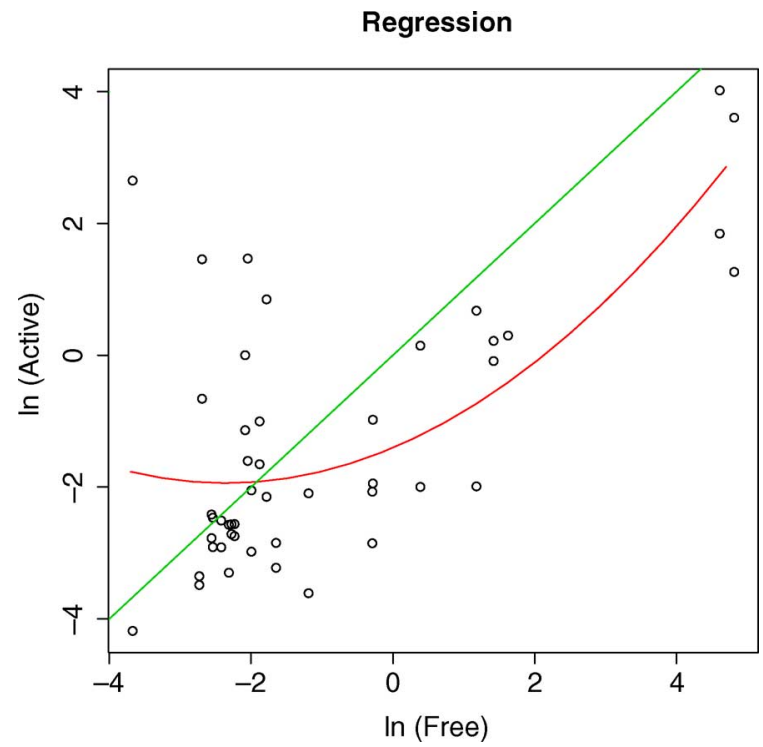

Fig. 10. Polynomial fitting to the data.

TABLE I

USERS INFORMATION

\begin{tabular}{cclll}
\hline & Estimate & $\begin{array}{l}\text { Std. } \\
\text { Error }\end{array}$ & t value & $P_{r}(>|t|)$ \\
\hline Intercept & -1.40 & 0.36 & -3.87 & 0.0003 \\
$\mathrm{X}$ & 0.45 & 0.11 & 4.21 & 0.00012 \\
$X^{2}$ & 0.09 & 0.04 & 2.34 & 0.024 \\
\hline
\end{tabular}

the adjusted $R^{2}$ of the fitting is 0.44 and all the coefficients of the fitting are statistically significant at 0.05 .

From this fitting it is possible to estimate where the orthosis suppress tremor efficiently, based on finding the points where the fitting crosses the line $y=x$ (green line on Fig. 10). This method allows us to identify a lower limit for efficient tremor suppression, this limit is roughly $0.15 \mathrm{rad}^{2} / \mathrm{s}^{3}$. In Fig. 9, we can check that the robotic exoskeleton has a minimum tremor 
TABLE II

RESULTS OF THE ANOVA ANALYSIS

\begin{tabular}{ccllllll}
\hline Source & & $\begin{array}{l}\text { Type III } \\
\text { Sum of } \\
\text { squares }\end{array}$ & df & $\begin{array}{l}\text { Mean } \\
\text { Square }\end{array}$ & Sig. \\
\hline Intercept & Hypothesis & 11.42 & 1.00 & 11.42 & 0.42 & 0.56 \\
& Error & 111.03 & 4.03 & 27.56 & & \\
\hline Mode & Hypothesis & 21.10 & 1.00 & 21.10 & 68.46 & 0.0 \\
& Error & 2.54 & 8.24 & 0.31 & & \\
\hline User & Hypothesis & 126.25 & 4.00 & 31.56 & 130.05 & 0.0 \\
& Error & 0.97 & 4.00 & 0.24 & & \\
\hline Mode * & Hypothesis & 0.97 & 4.00 & 0.24 & 0.32 & 0.86 \\
User & Error & 19.50 & 26.00 & 0.75 & & \\
\hline
\end{tabular}

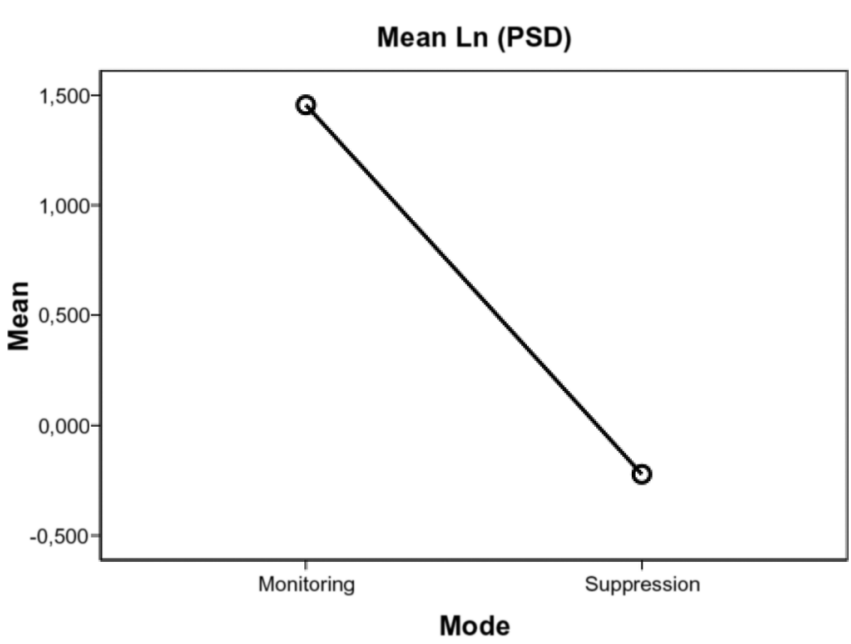

Fig. 11. Mode effect on the $\operatorname{Ln}(\mathrm{PSD})$.

suppression limit, i.e., if the spectral density of tremor movement is below the lower limit identified, around $0.15 \mathrm{rad}^{2} / \mathrm{s}^{3}$, WOTAS exoskeleton is ineffective in suppressing the tremor.

In order to verify the hypothesis that over the lower limit $\left(0.15 \mathrm{rad}^{2} / \mathrm{s}^{3}\right)$ WOTAS is efficient in tremor suppression, an univariate analysis of variance (ANOVA) analysis was performed. This analysis was made for all movements which tremor in free mode was above the threshold obtained by the regression curve. The hypothesis is that the orthoses is efficient for severe cases of tremor, when tremor is small (under the threshold of $0.15 \mathrm{rad}^{2} / \mathrm{s}^{3}$ ) the system has a negligible contribution. Five from the six users had movements in which the PSD of tremor was above the threshold and these are the measurements included in the analysis. The analysis was based on two factors: system in monitoring mode and system in suppression mode. Users were included to the model and the dependent variable was the Neperian Logarithm of the power spectral density $-\operatorname{Ln}(\mathrm{PSD})$. The results of this ANOVA analysis are shown in Table II. According to the table, the effects due to the mode (active versus monitoring) as well as due to the user are statistically significant. Moreover, as we can see in Fig. 11, the active mode reduces the mean value of the $\operatorname{Ln}(\mathrm{PSD})$.

The user also have a significant effect on the Ln(PSD) but not on the interaction mode-user as showed in Table II. However, WOTAS demonstrated its effect in reducing the tremor component in all users with tremor superior to the threshold (Fig. 12).

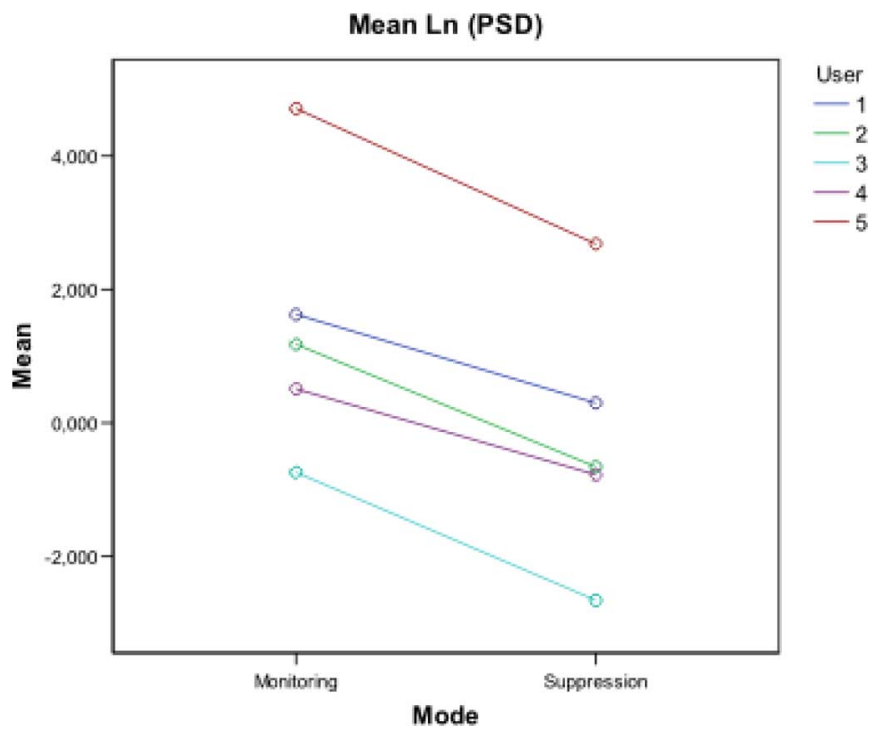

Fig. 12. Effect of the user in the reduction of tremor with WOTAS.
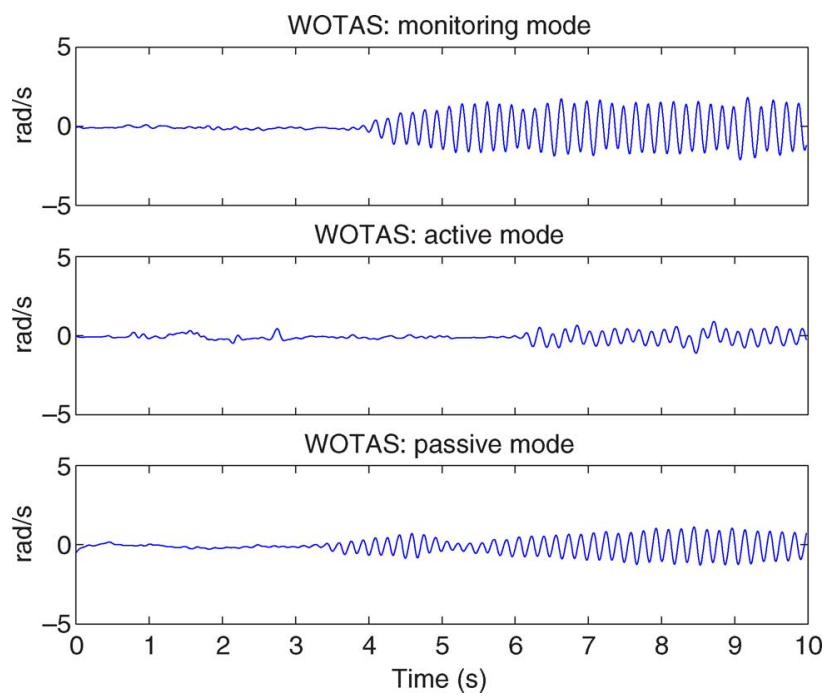

Fig. 13. This figure illustrates the oscillations of the elbow of tremor with WOTAS in a monitoring mode and suppressing modes in user 2. Note the strong reduction in amplitude of tremor when suppressing actions are applied. Oscillations are expressed in $\mathrm{rad} / \mathrm{sec}$.

The results also indicated that the range of reduction in tremor energy for signals above this orthosis operational limit ranges from $3.4 \%$ (percentile 5) to $95.2 \%$ (percentile 95) in relation to energy in monitoring mode.

These reductions can be appreciated in Figs. 13 and 14. These figures illustrate the effects of WOTAS on tremorous movement using both strategies. Fig. 13 illustrates the time series corresponding to the tremorous movement of the elbow joint of user 2 while the arm is outstretched. The top part of the figure shows the time signal with WOTAS in the monitoring mode. Notice that for both passive and active modes the amplitude of tremor is clearly smaller than in the monitoring mode.

Fig. 14 illustrates the same reduction in the frequency domain. The power spectrum density (PSD) have been obtained from the part of the signal with tremor. The top part of the figure illustrates the PSD of the tremorous movement with WOTAS 


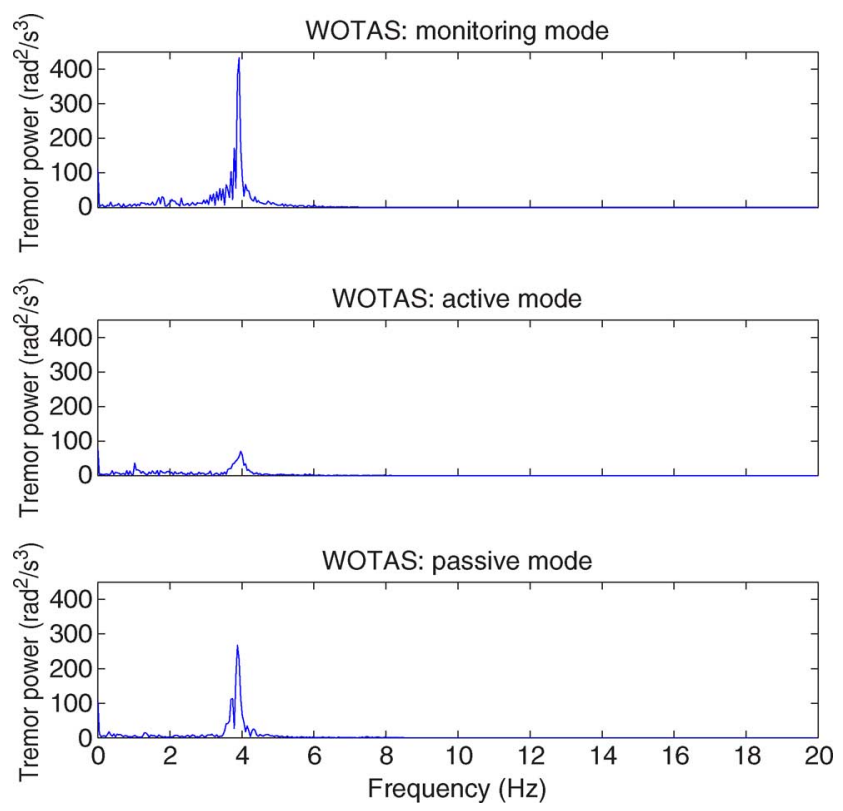

Fig. 14. This figure illustrates the associated power spectral density (PSD) of tremor WOTAS in a monitoring mode (upper part) and suppressing modes in user 2. Note the strong reduction in the PSD of tremor when suppressing actions are applied. PSD is expressed in $\mathrm{rad}^{2} / \mathrm{s}^{3}$.

operating in monitoring mode. It is possible to see a clear peak of tremor activity close to $4 \mathrm{~Hz}$. In the middle, figure shows the PSD while WOTAS was operating in active mode. Notice that the energy associated to tremor activity has been substantially reduced. In the low part of the figure the peak of energy corresponding to the tremorous activity when WOTAS is in passive mode also presents a clear reduction. These results indicate that WOTAS is able to suppress tremor in addition to validate both control strategies proposed, active and passive, for control of orthotic suppression of tremor. Notice that the frequency of tremor does not change when the exoskeleton is working in suppression modes.

A detailed analysis of the data showed that the active suppression strategy ( $81.2 \%$ mean power reduction) presents higher levels of tremor suppression compared to passive suppression strategy (70\% mean power reduction). This suggests a better performance of the active mode in tremor suppression.

\section{DISCUSSION}

The previous section presents results of the clinical trials related to the evaluation of the exoskeleton for tremor suppression presented in the paper. The exoskeleton performance was assessed in 10 users suffering from different kinds of tremors at the upper-limb. Two strategies for tremor reduction have been tested: viscous friction and notch filtering.

The analysis of the videos from the experiments demonstrated that in the majority of users there was no tremor displacement to proximal joints of the upper limb. Nevertheless, one user in ten presented an increment in tremor activity at shoulder level. The authors believe that future work should be performed in order to investigate and define the profile of the users affected by this phenomenon.
The results of the experiments indicated that the device could achieve a consistent $40 \%$ of tremor power reduction for all users, being able to attain a reduction ratio in the order of $80 \%$ tremor power in specific joints of users with severe tremor. In addition, the users reported that the exoskeleton did not affect their voluntary motion. These results indicate the feasibility of tremor suppression through biomechanical loading. Nevertheless, the users reported that the exoskeleton could not be considered as a solution to their problem since it is bulky and heavy. The users considered that the use of such device should cause social exclusion. This was expected since the exoskeleton was developed as a platform to evaluate the concept of mechanical tremor suppression and not as a final orthotic solution. The main wish expressed by the potential users was the possibility of hiding the exoskeleton under clothing [12].

The results also indicated a superior performance of active tremor suppression over passive tremor suppression. However, the authors believe that this could be due to the fact that the value of viscosity added to the movement was the same for all users. The customization of viscosity or inertia added to the upper limb according to the biomechanical characteristics of each user should improve the efficiency of passive tremor suppression strategy [12], [24].

It was noticed that the degree of tremor reduction was dependent upon the power associated with tremor. There are lower limits for robotic tremor suppression that should be determined for the mechanical impedance of the contact exoskeleton-skin, and the particular morphology of tremor.

During the trials two users spontaneously reported that they felt a decrease in the amplitude of their tremorous movement and consequently they felt more confident about the execution of the task. This indicates that the visual feedback of a smooth movement has a positive impact in the user. This fact is very important and future research will be performed in order to evaluate this phenomenon with more users of different pathologies.

\section{CONCLUSION}

This paper presented a robotic exoskeleton able to monitor, diagnose and control tremor in subjects. This robotic exoskeleton is equipped with kinematic and kinetic sensors for the measurement and calculation of joint angular displacement, velocity and acceleration, as well as interaction forces between the limb and orthosis. In addition, it could also apply dynamic force to the articulations of the upper limb by means of a set of flat dc motors in combination with pancake gears. The big innovations of the WOTAS exoskeleton are its portability, its non invasiveness, and that it provides direct information from each joint of the upper limb. The exoskeleton was evaluated with 10 users and validated the concept of tremor suppression through wearable robotics.

Both proposed strategies for tremor suppression (viscous friction and notch-filtering) have produced significant reduction of tremor amplitude. The results indicated that notch-filtering of tremor is more efficient than viscous friction, however further research should be performed in order to validate this statement.

Some aspects of mechanical tremor suppression were not evaluated in this study, further trials with a larger number of users should be held in order to evaluate other different aspects 
of WOTAS operation. The exoskeleton will help to analyze the effect of biomechanical loading on the upper limb motion and quantify its effects on fatigue. In addition, the carryover of attenuation effects after the impedance is removed must be subject to study.

Another very important issue is the interface of the exoskeleton with the upper limb. It was detected that there are lower limits for robotic tremor suppression determined by the mechanical impedance of the contact exoskeleton-upper limb. These limitations make the device useless for users with moderate to low tremor. There are several biomechanical aspects regarding the transmission of forces from the actuators to the limb that should be solved in order to improve the exoskeleton performance. Another aspect that requires further research is the effect of visual feedback on the capacity of the user to perform a task.

The capacity of applying internal dynamic forces to the upper limb opens widely the application field of WOTAS exoskeleton. It could be applied in different areas of the rehabilitation robotic field, for instance, it could provide restoration or maintenance of motor function to different joints of the upper limb. Most of the powered orthoses designed to date are nonambulatory devices [30]. There is a need in the rehabilitation area of ambulatory devices able to apply dynamic forces to the upper limb.

\section{REFERENCES}

[1] A. Anouti and W. Koller, "Tremor disorders: Diagnosis and management," Western J. Med., vol. 162, no. 6, pp. 523-530, 1998.

[2] R. Elble and W. Koller, Tremor. Baltimore, MD: Johns Hopkins Univ. Press, 1990.

[3] E. Rocon, J. Belda-Lois, J. Sanchez-Lacuesta, and J. L. Pons, "Pathological tremor management: Modelling, compensatory technology and evaluation,” Technol. Disability, vol. 16, pp. 3-18, 2004.

[4] J. Kotovsky and M. Rosen, "A wearable tremor-suppression orthosis," J. Rehabil. Res. Develop., vol. 35, no. 4, 1998.

[5] M. Rosen, A. Arnold, I. Baiges, M. Aisen, and S. Eglowstein, "Design of a controlled-energy-dissipation orthosis (cedo) for functional suppression of intention tremors," J. Rehabil. Res. Develop., vol. 32, no. 1, pp. 1-16, 1995.

[6] B. Adelstein, "Peripheral mechanical loading and the mechanism of abnormal intention tremor," M.S. thesis, Massachusetts Inst. Technol., Cambridge, 1981.

[7] M. Manto, M. Topping, M. Soede, J. Sanchez-Lacuesta, W. Harwin, J. Pons, J. Williams, S. Skararup, and L. Normie, "Dynamically responsive intervention for tremor suppression," IEEE Eng. Med. Biol., vol. 22, no. 3, pp. 120-132, May/Jun. 2003.

[8] E. Rocon, A. Ruiz, J. Pons, J. Belda-Lois, and J. Sánchez-Lacuesta, "Rehabilitation robotics: A wearable exo-skeleton for tremor assessment and suppression," in Int. Conf. Robot. Automat., 2005, pp. 241-246.

[9] Guides to the evaluation of permanent impaired Amer. Med. Assoc., 1993.

[10] I. Kapandji, The Physiology of the Joints: Upper Limb. New York: Churchill Livingstone, 1983, vol. 1.

[11] E. Rocon, J. Belda-Lois, J. Sánchez-Lacuesta, A. Ruiz, and J. Pons, "Estimation of biomechanical characteristics of tremorous movements based on gyroscopes," presented at the Conf. Asistive Technol.-From Virtuality to Reality, Lille, France, 2005.

[12] E. Rocon, "Reducción activa de temblor patológico de miembro superior mediante exoesqueletos robóticos," Ph.D. dissertation, Univ. Politécnica Madrid, Madrid, Spain, 2006.

[13] K. Tong and M. H. Granat, "A practical gait analysis system using gyroscopes," Med. Eng. Phys., vol. 21, pp. 87-94, 1999.

[14] E. Rocon, A. Ruiz, and J. Pons, "On the use of rate gyroscopes for tremor sensing in the human upper limb," in Proc. Int. Conf. Eurosensors XIX, 2005, p. MP30.
[15] E. Rocon, A. F. Ruiz, and J. L. Pons, "On the use of ultrasonic motors in orthotic rehabilitation of pathologic tremor," in Proc. ACTUATOR 2004 Conf., 2004, pp. 387-390.

[16] A. F. R. Olaya, "Sistema háptico para monitorización e intervención en pacientes con temblor patológico," M.S. thesis, Escuela Técnica Superior de Telecomunicación, Univ. Politécnica Madrid, Madrid, Spain, 2005.

[17] E. Rocon, A. F. Ruiz, and J. L. Pons, "Biomechanical modelling of the upper limb for robotics-based orthotic tremor suppression," Appl. Bionics Biomechan., vol. 2, no. 2, pp. 81-85, 2005.

[18] C. Riviere, S. Reich, and N. Thakor, "Adaptive fourier modeling for quantification of tremor," J. Neurosci. Methods, vol. 74, pp. 7-87, 1997.

[19] C. Riviere, "Adaptive Suppression of Tremor for Improved HumanMachine Control," Ph.D. dissertation, Johns Hopkins Univ., Baltimore, MD, 1995.

[20] K. Mann, F. W. Werner, and A. K. Palmer, "Frequency spectrum analysis of wrist motion for activities of daily living," J. Orthopedic Res., vol. 7, no. 2, pp. 304-306, 1989.

[21] Y. Bar-Shalom and X. Li, Estimation and Tracking: Principles, Techniques, and Software. Norwood, MA: Artech House, 1998.

[22] E. Brookner, Tracking and Kalman Filtering Made Easy. New York: Wiley, 1998.

[23] N. Hogan, "Impedance control: An approach to manipulation: Part iiiand iii," J. Dynamics Syst., Measurem. Control, vol. 107, no. 1, pp. $1-23,1985$.

[24] S. Pledgie, K. E. Barner, S. K. Agrawal, and T. Rahman, "Tremor suppression through impedance control," IEEE Trans. Rehabil. Eng., vol. 8, no. 1, pp. 53-59, Mar. 2000.

[25] E. Rocon, A. F. Ruiz, and J. Pons, "Theoretical control discussion on tremor suppression via biomechanical loading," in Proc. AAATE2003 Conf.-Assistive Technol.-Shaping Future, 2003, pp. 832-836.

[26] T. Inoue, "Practical repetitive control system design," in Proc. 29th IEEE Conf. Decision Control, 1990, vol. 3, pp. 1673-1678.

[27] N. Tsagarakis and D. Caldwell, "Development and control of a soft-actuated exoskeleton for use in physiotherapy and training," Autonomous Robots, vol. 15, pp. 21-33, 2003.

[28] J. Belda-Lois, M. Vivas, A. Castillo, F. Peydro, J. Garrido, J. SanchezLacuesta, R. B. R, R. Poveda, and J. Prat, "Functional assessment of tremor in the upper-limb," in Proc. 8th Congress Eur. Federation Res. Rehabil., 2004, p. MP30.

[29] R Development Core Team, R: A language and environment for statistical computing R Foundation Stat. Comput., Vienna, Austria, 2006 [Online]. Available: http://www.R-project.org

[30] G. Johnson, D. Carus, G. Parrini, S. S. Marchese, and R. Valeggi, "The design of a five degree-of-freedom powered orthosis for the upper limb," in Proc. Instrum. Mech. Eng., 2002, vol. 25, p. H.

E. Rocon, photograph and biography not available at the time of publication.

J. M. Belda-Lois, photograph and biography not available at the time of publication.

A. F. Ruiz, photograph and biography not available at the time of publication.

M. Manto, photograph and biography not available at the time of publication.

J. C. Moreno, photograph and biography not available at the time of publication.

J. L. Pons, photograph and biography not available at the time of publication. 